\title{
Psicooncología
}

ISSN: 1696-7240

\section{Influencia del optimismo y la percepción de apoyo social del personal sanitario sobre la calidad de vida de los enfermos de cáncer}

\author{
Iván Ruiz-Rodríguez ${ }^{1 *}$; Isabel Hombrados-Mendieta²; Anabel Melguizo-Garín ${ }^{3}$
}

Recibido: 14 de julio de 2020 / Aceptado: 1 de marzo de 2021

Resumen. Objetivo: El presente estudio se plantea analizar la relación del apoyo social proporcionado por el personal sanitario y el optimismo del paciente con la calidad de vida y el estrés percibido por los enfermos de cáncer. Método: Se recogen datos referidos a características sociodemográficas, de salud, optimismo, apoyo social, calidad de vida y estrés percibido de 200 pacientes de cáncer con una edad media de 50,5 años (DT = 13,05). El 73,5\% son mujeres y el 26,5\% son hombres. Resultados: La satisfacción con el apoyo recibido del personal sanitario (emocional, instrumental e informacional) y el optimismo se relacionan con una mayor calidad de vida y un menor estrés percibido. Los modelos predictivos indican que la satisfacción con el apoyo instrumental es el que más disminuye el estrés de los pacientes. Respecto a la calidad de vida los modelos predictivos indican que es el apoyo informacional el que más aumenta el estado global de salud, el apoyo emocional y el instrumental los que mejoran el funcionamiento y el apoyo instrumental es el que más reduce los síntomas de los pacientes. También se han encontrado diferencias de género, las mujeres perciben significativamente más estrés y peor calidad de vida que los hombres. Conclusión: El personal sanitario es una fuente de apoyo útil para las personas con cáncer y el optimismo mejora la calidad de vida de los pacientes oncológicos. De este estudio se resaltan importantes implicaciones prácticas.

Palabras clave: apoyo social, optimismo, calidad de vida, estrés percibido, personal sanitario, cáncer.

\section{[en] Influence of optimism and perception of social support from health personnel on the quality of life of cancer patients}

Abstract: Objective: The present study aims to analyze the relationship of social support provided by health personnel and the patient's optimism with quality of life and stress perceived by cancer patients. Method: Data regarding sociodemographic characteristics, health, optimism, social support, quality of life and perceived stress are collected from 200 cancer patients with a mean age of 50.5 years $(\mathrm{SD}=13.05) .73 .5 \%$ are women and $26.5 \%$ are men. Results: Satisfaction with the support received

1 Iván Ruiz-Rodríguez. Departamento de Psicología Social. Facultad de Estudios Sociales y del Trabajo. Universidad de Málaga. Málaga (España)

E-Mail: ivan_dlr@uma.es

2 Isabel Hombrados-Mendieta. Departamento de Psicología Social. Facultad de Estudios Sociales y del Trabajo. Universidad de Málaga. Málaga (España).

E-Mail: mihombrados@uma.es

3 Anabel Melguizo-Garín. Departamento de Psicología Social. Facultad de Estudios Sociales y del Trabajo. Universidad de Málaga. Málaga (España)

E-Mail: anamel@uma.es

* Dirección de correspondencia: Iván Ruiz-Rodríguez. Departamento de Psicología Social. Facultad de Estudios Sociales y del Trabajo. Universidad de Málaga. Avenida Francisco Trujillo Villanueva s/n. 29071 Málaga (España). E-Mail: ivan_dlr@uma.es 
from health personnel (emotional, instrumental and informational) and optimism are related to a higher quality of life and less perceived stress. Predictive models indicate that satisfaction with instrumental support is the one that most reduces the stress of patients. Regarding quality of life, predictive models indicate that informational support is the one that most increases the global health status, emotional and instrumental supports that improve functioning, and instrumental support is the one that most reduces patients' symptoms. Gender differences have also been found, women perceive significantly more stress and worse quality of life than men. Conclusion: Health personnel are a useful source of support for people with cancer and optimism improves the quality of life for cancer patients. This study highlights important practical implications.

Keywords: social support, optimism, quality of life, perceived stress, health personnel, cancer.

Sumario: 1. Introducción 2. Método 3. Análisis de datos 4. Resultado 5. Discusión y conclusión. 6. Agradecimientos 7. Referencias bibliográficas

Cómo citar: Ruiz-Rodríguez I, Hombrados-Mendieta I, Melguizo-Garín A. Influencia del optimismo y la percepción de apoyo social del personal sanitario sobre la calidad de vida de los enfermos de cáncer. Psicooncología 2021; 18: 51-75. doi: 10.5209/psic.74532

\section{Introducción}

En la actualidad el cáncer es una de las principales causas de muerte en la población. En 2018 fue el responsable de aproximadamente 9,6 millones de muertes (casi 1 de cada 6 muertes), siendo la segunda causa de muerte a nivel mundial ${ }^{(1)}$. En Europa se producen más de 3 millones de casos nuevos de cáncer y 1,7 millones de muertes cada año, aproximadamente el 20\% de las muertes en Europa ${ }^{(2)}$. En España, en 2018 el cáncer fue la segunda causa de muerte tras las enfermedades del sistema circulatorio, provocando 112.714 muertes, el $26,4 \%$ del total de defunciones en España, un $2,2 \%$ más que en $2017^{(3)}$. A pesar del aumento del diagnóstico precoz, los avances terapéuticos y el incremento de supervivientes de cáncer, puede llegar a ser una enfermedad crónica que ocasiona que el paciente y sus familiares tengan que hacer frente a múltiples situaciones y eventos estresantes ${ }^{(4)}$. El cáncer puede conllevar un deterioro de la calidad de vida ${ }^{(5)}$ ya que suele deteriorar ámbitos de la vida personal, familiar, laboral y social del paciente durante largos intervalos de tiempo.

\section{Calidad de vida}

La calidad de vida es un concepto multidimensional que se define como el grado en que el bienestar físico, funcional, social o emocional esperado por el paciente se ve disminuido por el proceso médico o por el tratamiento ${ }^{(6)}$. El cáncer merma físicamente y reduce notablemente la calidad de vida los pacientes ${ }^{(7)}$. Existen multitud de factores que influyen en la reducción de la calidad de vida de pacientes con cáncer: El tipo de cáncer, el tipo de tratamiento y los efectos secundarios del tratamiento como son la fatiga, las náuseas y los vómitos, ansiedad o depresión ${ }^{(7,8)}$. Esta disminución de la calidad de vida parece ser un resultado común observado en pacientes con todo tipo de cáncer ${ }^{(9,10)}$ así como en pacientes que se encuentran en cualquier fase del proceso sanitario $^{(11)}$. No solo la calidad de vida merma en pacientes que se encuentran en tratamiento, también se reduce en pacientes libres de enfermedad, que se encuentran en fase de revisión, por el miedo y la preocupación de la recurrencia ${ }^{(12)}$. Entre los 
factores que influyen en la calidad de vida se han encontrado algunos factores sociodemográficos como el género. Las mujeres con cáncer suelen percibir menos calidad de vida que los hombres ${ }^{(13)}$. Una posible explicación es que las mujeres y los hombres tienen diagnósticos de diferentes tipos de cáncer, lo que puede influir en esa diferencia de impacto de la enfermedad en la calidad de $\operatorname{vida}^{(14)}$. A ello hay que añadir el hecho de que las mujeres tienen una esperanza de vida mayor que los hombres lo que significa que van a pasar más tiempo con miedo a la posible recidiva y un mayor tiempo donde la enfermedad va reduciendo la calidad de vida del paciente.

La evaluación de la calidad de vida es relevante para planificar el tratamiento de los pacientes llegando a ser un factor pronóstico del tratamiento ${ }^{(15)}$. De hecho, muchos pacientes con cáncer asumen el riesgo de los diferentes tratamientos solo si conocen cómo les va a influir en su calidad de vida, ya que consideran su calidad de vida tan importante como su cantidad de vida ${ }^{(16)}$.

\section{Estrés percibido}

El cáncer hace que el paciente se tenga que enfrentar a múltiples dificultades y situaciones potenciales de estrés en cada fase del proceso sanitario, como son, la ruptura de relaciones, problemas financieros, fatiga y la interrupción del trabajo ${ }^{(17)}$. Por ello el aumento del estrés es una de las consecuencias más comunes entre los pacientes con cáncer y se relaciona con el aumento de síntomas y problemas psicológicos ${ }^{(18)}$. El diagnóstico produce un elevado estrés ya que crea en el paciente una incertidumbre sobre su futuro debido a lo traumática e inesperada que es la experiencia ${ }^{(19)}$. Otro factor que contribuye al aumento del estrés es el tratamiento en sí mismo ${ }^{(20)}$. Incluso después de finalizar el tratamiento pueden continuar existiendo factores que aumenten el estrés percibido por el paciente como son los problemas de salud derivados del tratamiento ${ }^{(21)} \mathrm{o}$ el miedo a la posible recidiva ${ }^{(22)}$ que se ha asociado con una reducción del bienestar en sobrevivientes de cáncer ${ }^{(23)}$. Las percepciones subjetivas del impacto de la enfermedad sobre la vida y el miedo al futuro están más asociados con el estrés que las propias características objetivas de la enfermedad ${ }^{(24)}$.

Por todo ello es importante tener en cuenta el estrés percibido por los pacientes con cáncer ya que parece estar asociado a su evaluación y capacidad de manejo de la calidad de vida ${ }^{(25)}$, pudiendo ser un predictor de una futura disminución del funcionamiento y salud en los pacientes ${ }^{(26)}$.

\section{Optimismo, calidad de vida y estrés percibido}

El optimismo disposicional hace referencia al grado en que una persona tiene expectativas favorables generalizadas respecto a su futuro. Es decir, una tendencia general a esperar resultados positivos ${ }^{(27)}$. Es un factor protector o amortiguador en pacientes con cáncer $^{(28)}$ que ayuda a los pacientes a sobrellevar mejor la enfermedad ${ }^{(29)}$ y favorece a la persona en la adaptación a la enfermedad, en la mejora de la calidad de vida y disminución del miedo y ansiedad, llegando incluso a tolerar mejor el tratamiento ${ }^{(30)}$. De hecho, un bajo optimismo disposicional pronostica los síntomas de ansiedad y depresión lo que conlleva una peor calidad de $\operatorname{vida}^{(31)}$. En pacientes con cáncer, el optimismo puede beneficiar la calidad del sueño ${ }^{(32)}$ asociándose el optimismo con la disminución de los síntomas de insomnio ${ }^{(33)}$. Los pacientes optimistas pueden responder tanto al diagnóstico como 
al tratamiento con espíritu de lucha, teniendo un efecto positivo en su calidad de vida, y los pacientes pesimistas responden con desesperanza lo que tiene un efecto negativo en su calidad de vida $^{(34)}$. Por ello los pacientes que mantienen el optimismo durante todo el proceso de la enfermedad son las personas que se adaptan mejor a ella, minimizando el impacto de la enfermedad en la calidad de vida. El optimismo también se asocia con una reducción del estrés percibido por el paciente ${ }^{(35)}$. Mejora las habilidades personales necesarias para hacer frente a las situaciones de estrés, y es una herramienta útil en la reducción del impacto de los eventos estresantes de los pacientes ${ }^{(36)}$. Llega a ser un factor clave para minimizar el impacto del estrés incluso en el sistema inmune de la persona ${ }^{(37)}$.

\section{Apoyo social, calidad de vida y estrés percibido}

Un fenómeno observado es la influencia del apoyo social en la mejora de la calidad de vida y en la reducción del estrés percibido por el paciente con cáncer ${ }^{(38,39)}$ y sus familiares ${ }^{(40,41)}$. El apoyo social es un concepto interactivo, una transacción interpersonal que se da entre las fuentes de apoyo y el receptor de ayuda. Conlleva emociones, ayuda material e información y se da en un contexto determinado. Este apoyo lo suelen proporcionar la comunidad, sus redes sociales y las relaciones de confianza e íntimas en situaciones cotidianas y en situaciones de crisis durante la $\mathrm{vida}^{(42)}$. Los autores, en su mayoría, destacan tres tipos de apoyo social(43): El apoyo emocional hace referencia a la seguridad de confiar en alguien y ser amado; El apoyo instrumental se refiere a tener disponible ayuda directa; y el apoyo informacional que incluye recibir información o consejo ${ }^{(44)}$. La satisfacción con el apoyo está determinada en gran medida por las necesidades del paciente, por lo que cada tipo de apoyo cumple una función específica ${ }^{(45)}$.

El apoyo social recibido no es el único factor relevante, además la fuente que proporciona el apoyo es fundamental para que el paciente perciba positivamente el apoyo recibido ${ }^{(46)}$. Una fuente de apoyo muy importante y poco estudiada es el personal sanitario. Esta fuente es muy relevante ya que tiene contacto con el paciente en todas las fases del proceso sanitario, desde el inicio del proceso sanitario en la fase de diagnóstico, pasando por el tratamiento, hasta las revisiones y el alta médica. En todas las fases acompaña al paciente y participa activamente. Las percepciones del paciente sobre la gravedad de la enfermedad y la amenaza que conlleva puede estar influidas en parte por la información del personal sanitario ${ }^{(47)}$. Si esta información es adecuada y los pacientes tienen la oportunidad de resolver dudas con el personal sanitario tiene un gran efecto en la calidad de vida de los pacientes ${ }^{(48)}$. De este modo, la información proporcionada por el personal sanitario contribuye a un mejor ajuste a la enfermedad por parte del paciente ${ }^{(49)}$. Hay que tener en cuenta que todos los pacientes mantienen contacto con el personal sanitario pero puede suceder que un paciente con alto deseo de apoyo social, no reciba el apoyo deseado y perciban un estrés más elevado ${ }^{(50)}$. También la necesidad de apoyo varía en cada etapa de la enfermedad, tanto en el tipo de apoyo que se necesita como en la red de apoyo que lo suministra. Esto puede provocar que el paciente perciba como poco útil determinado apoyo proporcionado en un momento determinado ${ }^{(43)}$. La satisfacción con el apoyo social recibido del personal sanitario no se ha estudiado en la mayoría de las investigaciones y la mayoría de los estudios no diferencian entre las dimensiones de apoyo y la satisfacción con éste ${ }^{(51)}$. 


\section{El presente estudio}

El objetivo de este estudio es analizar cómo influyen el apoyo social y el optimismo en la calidad de vida y el estrés percibido en los enfermos oncológicos atendiendo a las diferentes fases de la enfermedad, los tipos de tratamiento y las diferencias de género. Una de las aportaciones de este estudio es que realiza un análisis de la satisfacción que tienen los pacientes oncológicos con los diferentes tipos del apoyo social (emocional, instrumental e informacional) proporcionados por el personal sanitario, que, a pesar de ser una fuente de apoyo fundamental, se carecen de estudios que expliquen cómo el apoyo de esta fuente influye en la calidad de vida y el estrés de los pacientes. Este análisis servirá para conocer cómo dos variables de protección frente al estrés, una de interacción social, como el apoyo social y otra personal, el optimismo, inciden en la calidad de vida y en la disminución del estrés percibido por los pacientes. Se carecen de estudios que analicen conjuntamente ambas variables, por ello este estudio proporciona un conocimiento más profundo sobre las variables que mejoran la calidad de vida de los pacientes oncológicos.

Las hipótesis planteadas son:

1) Las mujeres con cáncer perciben peor calidad de vida y estrés que los hombres.

2) Las personas que están en tratamiento perciben más estrés y tienen peor calidad de vida que los que se encuentran en revisión

3) La satisfacción con el apoyo emocional, instrumental e informacional proporcionado por el personal sanitario y el optimismo están relacionados positivamente con la calidad de vida y negativamente con el estrés percibido de los pacientes con cáncer.

\section{Método}

\section{Muestra}

Los participantes en este estudio fueron 200 pacientes con cáncer con edades comprendidas entre los 22 y los 88 años, siendo la medida de edad total 50,55 (DT = $13,05) .147$ pacientes son mujeres $(73,5 \%)$ y 53 son hombres $(26,5 \%)$. Los pacientes han tenido diferentes tipos de cáncer y se encuentran en diferentes fases del proceso sanitario (tabla 1). Los pacientes proceden de diferentes asociaciones y del Hospital Costa del Sol (Marbella) donde acudían a revisión o a recibir tratamiento. Los criterios de inclusión fueron: personas con diferentes tipos de cáncer, mayores de edad, que estén en la actualidad en tratamiento o en la fase de revisión. Los criterios de exclusión fueron expacientes de cáncer que hubiesen concluido la fase de revisión o seguimiento y jóvenes con cáncer menores de edad. 
Tabla 1. Variables sociodemográficas y de salud $(n=200)$

\begin{tabular}{|c|c|}
\hline Edad & $50,55(13,05)^{\mathrm{a}}$ \\
\hline Género & $\%(\mathrm{~N})$ \\
\hline Hombre & $26,5(53)$ \\
\hline Mujer & $73,5(147)$ \\
\hline \multicolumn{2}{|l|}{ Estado civil } \\
\hline Soltero/a & $16,0(32)$ \\
\hline Casado/a & $64,0(128)$ \\
\hline Divorciado/a / Separado/a & $7,5(15)$ \\
\hline Viudo/a & $4,5(9)$ \\
\hline Pareja de hecho & $8,0(16)$ \\
\hline \multicolumn{2}{|l|}{ Nivel académico } \\
\hline Universitarios o superiores terminados & $35,5(71)$ \\
\hline Ahora en estudios universitarios o superiores & $3,5(7)$ \\
\hline Bachillerato o ciclo profesional terminado & $27,5(55)$ \\
\hline Enseñanza obligatoria (antes EGB) terminada & $28,0(56)$ \\
\hline Sin ninguno de los estudios anteriores & $5,5(11)$ \\
\hline \multicolumn{2}{|l|}{ Fase del proceso médico } \\
\hline En tratamiento & $40,5(81)$ \\
\hline En revisión & $59,5(119)$ \\
\hline \multicolumn{2}{|l|}{ Tipo de cáncer } \\
\hline Tiroides & $32,1(63)$ \\
\hline Mama & $28,6(56)$ \\
\hline Laringe & $9,2(18)$ \\
\hline Colon & $7,1(14)$ \\
\hline Ovario & $6,6(13)$ \\
\hline Pulmón & $3,1(6)$ \\
\hline Otro & $13,3(26)$ \\
\hline \multicolumn{2}{|l|}{ Tratamiento quimioterapia } \\
\hline $\mathrm{Si}$ & $58,3(116)$ \\
\hline No & $41,7(83)$ \\
\hline \multicolumn{2}{|l|}{ Tratamiento radioterapia } \\
\hline $\mathrm{Si}$ & $56,8(113)$ \\
\hline No & $43,2(86)$ \\
\hline \multicolumn{2}{|l|}{ Tratamiento quirúrgico } \\
\hline $\mathrm{Si}$ & $87,4(174)$ \\
\hline No & $12,6(25)$ \\
\hline \multicolumn{2}{|l|}{ Tratamiento hormonoterapia } \\
\hline $\mathrm{Si}$ & $21,1(42)$ \\
\hline No & $78,9(157)$ \\
\hline \multicolumn{2}{|l|}{ Tiempo desde el diagnóstico inicial } \\
\hline Menos de 1 año & $25,5(51)$ \\
\hline 1-2 años & $13,0(26)$ \\
\hline 2-5 años & $37,0(74)$ \\
\hline Más de 5 años & $23,0(46)$ \\
\hline
\end{tabular}

a Media (Desviación Típica) 


\section{Diseño}

Se trata de un estudio transversal correlacional mediante metodología de encuesta por autoinforme.

\section{Variables e instrumentos}

Calidad de Vida: EORTC-QLQ C-30-Organización Europea para la Investigación y Tratamiento del Cáncer(52). Usamos el cuestionario adaptado a la población española. Este cuestionario identifica los aspectos valorativos de diferentes áreas de la calidad de vida, informados por el paciente con cáncer ${ }^{(53)}$. Está formado por 28 ítems tipo escala Likert con puntuación entre 1 y 4, desde "En absoluto" hasta "Mucho" y dos ítems tipo escala Likert con puntuación entre 1 y 7, desde "Pésima" hasta "Excelente". El cuestionario tiene una escala global de salud, 5 escalas funcionales: física (p. ej. ¿tiene alguna dificultad para dar un paseo largo?), de rol (p. ej. ¿ha tenido algún impedimento para hacer su trabajo u otras actividades cotidianas?), emocional (p. ej. ¿se sintió preocupado?), cognitiva (p. ej. ¿ha tenido dificultades para recordar cosas?) y social (p. ej. ¿ha interferido su estado físico o el tratamiento médico en sus actividades sociales?), 3 escalas de síntomas (fatiga, nauseas/vómitos y dolor) y 6 ítems (disnea, pérdida de apetito, insomnio, estreñimiento, diarrea y dificultades económicas). Las puntuaciones se transforman en una puntuación lineal en escala de 0-100 según el manual de la EORTC. Una puntuación alta para el estado global de salud y las escalas funcionales representa una alta calidad de vida. Una puntuación alta para la escala de síntomas representa una baja calidad de vida. Esta escala muestra una fiabilidad Alpha de Cronbach $\alpha=0,86$.

Apoyo Social: Cuestionario de Frecuencia y Satisfacción con el Apoyo Social $(\text { QFSSS })^{(54)}$. Mide el apoyo social desde una perspectiva multidimensional, diferenciando los tipos de apoyo (emocional, instrumental e informacional) que proporcionan cada una de las fuentes de apoyo. En el estudio se incluyó como fuente de apoyo el personal sanitario para evaluar la satisfacción con el apoyo emocional, instrumental e informacional proporcionado por esta fuente de apoyo. Tal y como señalan los autores del instrumento, se pueden incorporar diferentes fuentes de apoyo en función del contexto que se vaya a analizar. Este instrumento ha sido empleado con diferentes fuentes de apoyo para diferentes poblaciones, como adolescentes ${ }^{(55)}$; inmigrantes ${ }^{(56)}$ y familiares oncológicos ${ }^{(40)}$ mostrando altos índices de fiabilidad.

Se utiliza una escala tipo Likert de 1 a 5, para evaluar la satisfacción con el apoyo recibido ( 1 = Insatisfecho y $5=$ Muy satisfecho). El Alpha de Cronbach de la escala completa es $\alpha=0,96$.

Estrés percibido: Escala de estrés percibido $(P S S)^{(57)}$. Usamos la versión adaptada a la población española ${ }^{(58)}$. Contiene 14 ítems con respuesta tipo Likert de 0 a 4 (0 = Nunca y 4 = Muy a menudo). Mide el grado en que las situaciones de la vida en el último mes son evaluadas como estresantes. Evalúa el grado en que las personas perciben que su vida es impredecible, incontrolable o está sobrecargada. Una mayor puntuación corresponde a un mayor nivel de estrés percibido. La consistencia interna del instrumento es de $\alpha=0,83$.

Optimismo disposicional: Test de Orientación Vital Revisado (LOT-R) ${ }^{(59)}$. Usamos la versión adaptada a la población española ${ }^{(60)}$. Contiene 6 ítems (más 4 ítems distractores) en escala tipo Likert de 5 puntos. 3 están redactados en positivo (optimismo) y 3 en 
negativo (pesimismo). Estos 3 últimos se revierten para obtener una puntuación final orientada al optimismo. La consistencia interna del instrumento es de $\alpha=0,78$.

\section{Procedimiento}

El estudio se realiza en el ámbito de las asociaciones y en el ámbito hospitalario. Participaron veinticinco asociaciones de pacientes de cáncer de 29 ciudades españolas. El cuestionario estaba disponible a través de una página web para que pudieran participar pacientes de diferentes ciudades. También se contactó con el equipo de oncología del Hospital Costa del Sol de Marbella. Para poder realizar las entrevistas de forma presencial a los pacientes que se encontraban en el Hospital, el estudio tuvo que ser aprobado por el Comité Ético del Hospital. La entrevista con los pacientes se realizaba una vez terminaban la consulta médica. Antes de comenzar la entrevista con los pacientes se explicaba a los participantes los objetivos de la investigación (a las personas que se encontraban fuera de la ciudad y lo realizaban a través de internet se les explicaba en la página web), asegurándoles la confidencialidad y anonimato de la participación mediante un documento de consentimiento informado.

El Comité Ético de la Universidad de Málaga, así como el del Hospital Costa del Sol dictaminaron que el estudio cumplía con criterios metodológicos éticos y legales que permitían su aplicación.

\section{Análisis de datos}

El análisis de datos se realizó con IBM SPSS versión 23. Se usaron estadísticos descriptivos para analizar las variables sociodemográficas y de salud de los participantes.

El coeficiente de correlación de Pearson se usó para comprobar la relación entre variables cuantitativas, como son el optimismo y la satisfacción con el apoyo social percibido del personal sanitario (emocional, instrumental e informacional) con la calidad de vida (estado global de salud, escalas de funcionamiento y escalas / ítems de síntomas) y el estrés percibido.

Las variables que en el análisis de correlación resultaron significativamente relacionadas con alguna dimensión de la calidad de vida y el estrés percibido fueron incluidas como variables independientes en el análisis de la regresión lineal múltiple. Las variables dependientes son cada una de las escalas/ítem de calidad de vida y de estrés percibido.

Para comparar las medias de las diferentes variables cuantitativas en función del género y de los diferentes tratamientos se realiza una prueba $t$ de Student para dos muestras independientes en cada una de las variables; género (hombre/mujer), fase del proceso médico (tratamiento/revisión), quimioterapia (sí/no), radioterapia (sí/ no), hormonoterapia (sí/no) y tratamiento quirúrgico (sí/no).

\section{Resultados}

En la tabla 2 se muestran los datos para la muestra total en calidad de vida, satisfacción con el apoyo social del personal sanitario, estrés percibido y optimismo disposicional. Los resultados muestran que las puntuaciones en las escalas/ítems son satisfactorias, 
existiendo unas altas puntuaciones en las escalas funcionales y la escala global de salud (siendo esta última más baja) y unas bajas puntuaciones en las escalas / ítems de síntomas. Las puntuaciones en satisfacción con el apoyo proporcionado del personal sanitario y las de optimismo disposicional son en general altas. En cambio, se hallan puntuaciones medias-bajas en el estrés percibido por los pacientes

Tabla 2. Media y Desviaciones Típicas de los Cuestionarios

\begin{tabular}{lc}
\hline EORTC QLQ-C30 & $\begin{array}{c}\text { Media } \\
\text { (Desviación Típica) }\end{array}$ \\
\hline Escala global de salud & $66,46(22,42)$ \\
\hline Escalas funcionales & \\
Funcionamiento físico & $83,90(16,94)$ \\
Funcionamiento de rol & $78,17(27,32)$ \\
Funcionamiento emocional & $70,83(25,19)$ \\
Funcionamiento cognitivo & $78,00(23,91)$ \\
Funcionamiento social & $72,33(28,82)$ \\
Escalas síntomas & \\
Fatiga & $34,00(27,19)$ \\
Náuseas y vómitos & $6,42(14,34)$ \\
Dolor & $24,33(25,88)$ \\
Disnea & $10,83(21,38)$ \\
Insomnio & $35,50(32,07)$ \\
Pérdida de apetito & $9,33(21,44)$ \\
Estreñimiento & $16,50(26,52)$ \\
Diarrea & $10,83(20,85)$ \\
Dificultades económicas & $22,33(31,19)$ \\
\hline CUESTIONARIO DE FECUENCIA Y SATSFACCÓn CON
\end{tabular}

CUESTIONARIO DE FRECUENCIA Y SATISFACCIÓN CON EL APOYO SOCIAL (QFSSS)

\begin{tabular}{lc}
\hline Satisfacción con apoyo emocional del personal sanitario & $3,81(1,42)$ \\
Satisfacción con apoyo instrumental del personal sanitario & $3,77(1,34)$ \\
Satisfacción con apoyo informacional del personal sanitario & $3,88(1,38)$ \\
\hline ESCALA DE ESTRÉS PERCIBIDO (PSS) & $38,63(16,21)$ \\
\hline TEST DE ORIENTACIÓN VITAL REVISADO (LOT-R) & $63,23(18,17)$ \\
\hline
\end{tabular}

\section{Diferencias de medias}

Las diferencias de medias del estrés percibido, escala global de salud y escalas funcionales en relación al género del paciente, la fase del proceso médico en la que se encuentran y el tipo de tratamiento recibido se muestran en la tabla 3. Las diferencias de medias de las escalas / ítems de síntomas en relación al género del paciente, la fase del proceso médico en la que se encuentran y el tipo de tratamiento recibido se muestran en tabla 4 . 


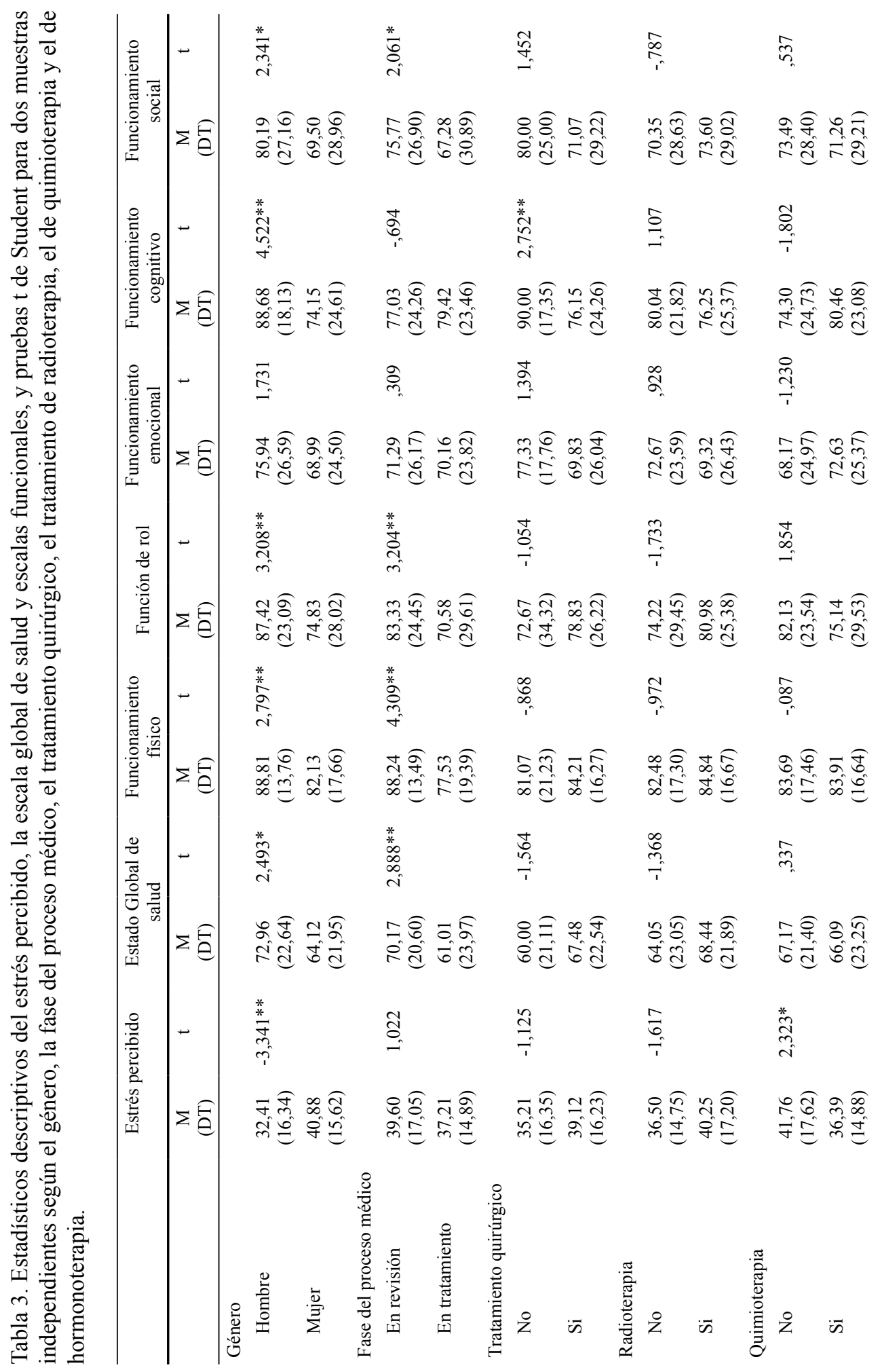




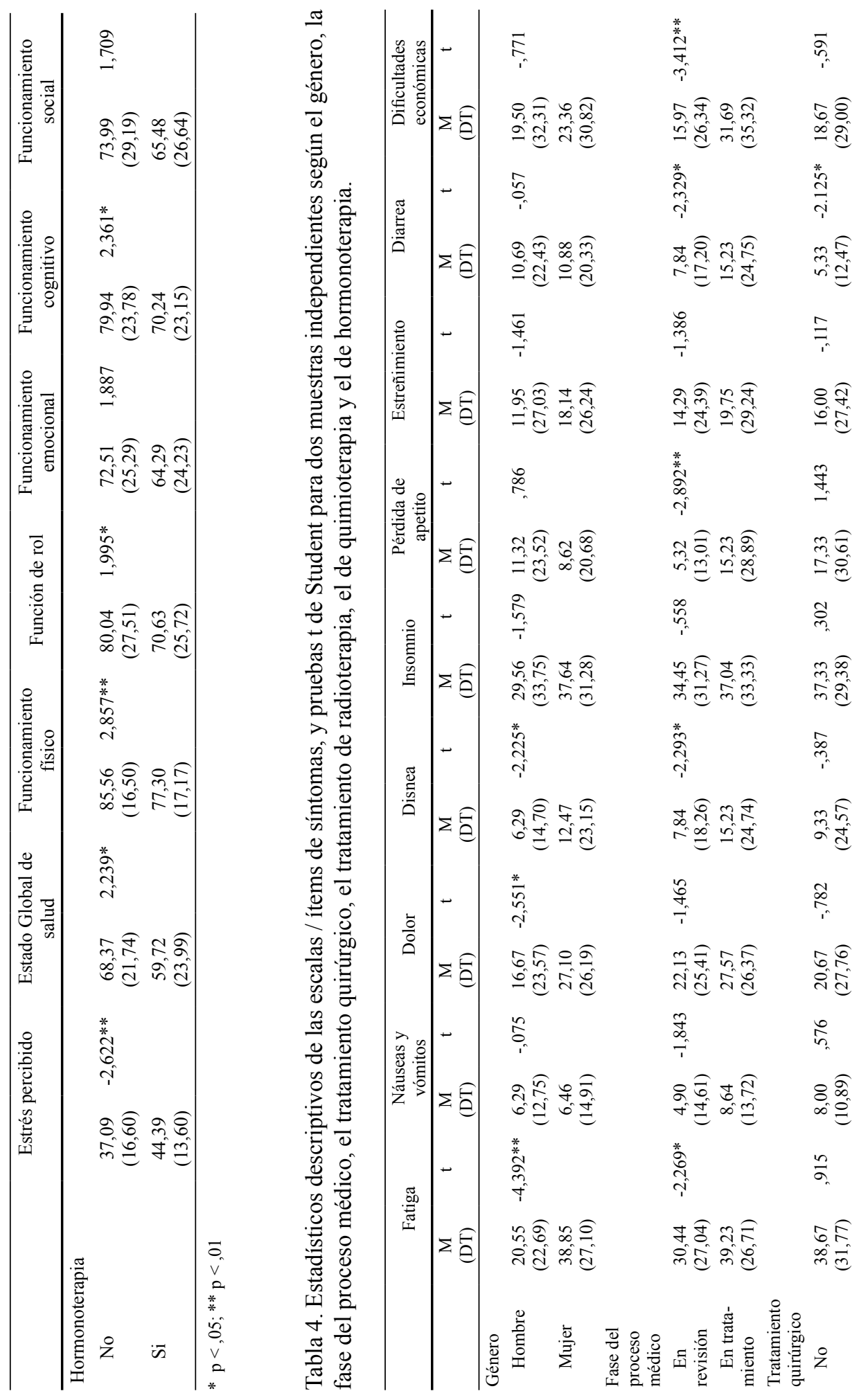




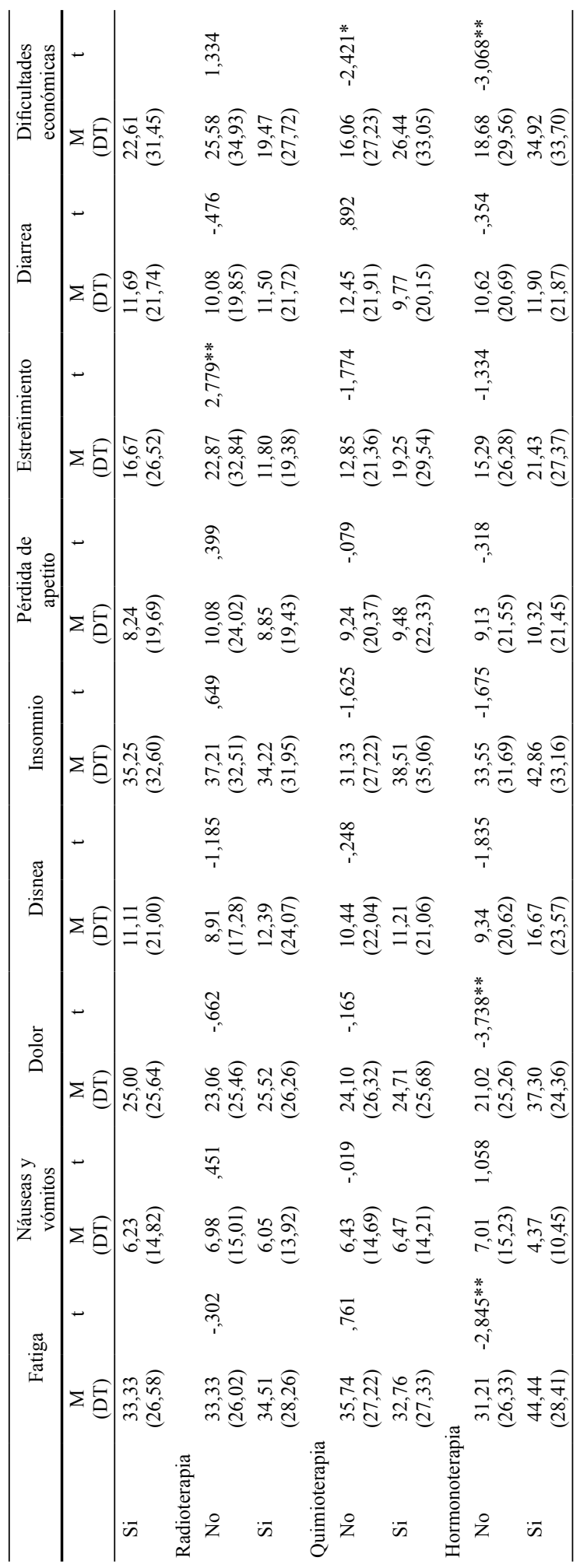


Encontramos diferencias estadísticamente significativas. Según los resultados obtenidos las mujeres perciben mayor estrés, un menor estado global de salud, menor funcionamiento físico, de rol, cognitivo y social, así como una mayor fatiga, dolor y disnea. Las personas que se encuentran en tratamiento actualmente muestran un peor estado global de salud, funcionamiento físico, de rol y social, así como más fatiga, disnea, pérdida de apetito, diarrea y mayores dificultades económicas. En relación a las condiciones de tratamiento encontramos diferencias significativas, de las cuales las más destacables son las encontradas respecto a la quimioterapia y hormonoterapia. Las personas que han recibido quimioterapia perciben menos estrés que las que no han recibido este tratamiento y muestran mayores dificultades económicas, mientras que las personas que han recibido hormonoterapia, muestran un mayor estrés percibido, un menor estado global de salud, funcionamiento físico, de rol y cognitivo y un mayor nivel de síntomas como fatiga y dolor, así como mayores dificultades económicas, que las personas que no han recibido este tratamiento.

Relación de la satisfacción con el apoyo social proporcionado por el personal sanitario y el optimismo con la calidad de vida y el estrés percibido de los pacientes.

En la Tabla 5 se muestran los coeficientes de correlación. El optimismo y la satisfacción con el apoyo social recibido que proporciona el personal sanitario (emocional, instrumental e informacional) se relacionan con una mejora del estado global de salud, del funcionamiento emocional, cognitivo y social, con una disminución de la fatiga y dolor y una disminución del estrés percibido. A su vez, el apoyo instrumental se relaciona con un mejor funcionamiento físico y el apoyo informacional con una disminución del síntoma de diarrea. El optimismo se relaciona con un mejor funcionamiento de rol y una disminución de la disnea y el insomnio. No encontramos relación entre optimismo y satisfacción con el apoyo social del personal sanitario en relación a algunos síntomas (náuseas y vómitos, pérdida de apetito y estreñimiento) ni respecto a las dificultades económicas.

Tabla 5. Matriz de correlaciones entre satisfacción con apoyo social y optimismo con calidad de vida y estrés percibido.

Satisfacción con apoyo del personal sanitario

Optimismo

Disposicional

\begin{tabular}{lcccc}
\hline EORTC QLQ-C30 & Emocional & Instrumental & Informacional & \\
\hline Estado global de salud &, $200^{* *}$ &, $249^{* *}$ &, $223^{* *}$ &, $271^{* *}$ \\
\hline Escalas funcionales & & & & \\
Funcionamiento físico &, 082 &, $128^{*}$ &, 104 &, 130 \\
Funcionamiento de rol &, 078 &, 090 &, 070 &, $171^{*}$ \\
Funcionamiento emocional &, $194^{* *}$ &, $204^{* *}$ &, $228^{* *}$ &, $408^{* *}$ \\
Funcionamiento cognitivo &, $307^{* *}$ &, $321^{* *}$ &, $321^{* *}$ &, $265^{* *}$ \\
Funcionamiento social &, $146^{*}$ &, $143^{*}$ &, $125^{*}$ &, $271^{* *}$ \\
\hline Escala síntomas & & & & \\
Fatiga &,$- 219^{* *}$ &,$- 261^{* *}$ &,$- 243^{* *}$ &,$- 277^{* *}$ \\
Náuseas y vómitos &, 017 &,- 010 &,- 016 &,- 106 \\
\hline
\end{tabular}




\begin{tabular}{lcccc}
\hline & \multicolumn{2}{c}{ Satisfacción con apoyo del personal sanitario } & $\begin{array}{c}\text { Optimismo } \\
\text { Disposicional }\end{array}$ \\
\hline EORTC QLQ-C30 & Emocional & Instrumental & Informacional & \\
\hline Dolor &,$- 135^{*}$ &,$- 167^{* *}$ &,$- 176^{* *}$ &,$- 162^{*}$ \\
Disnea &,- 057 &,- 081 &,- 115 &,$- 211^{* *}$ \\
Insomnio &,- 097 &,- 108 &,- 104 &,$- 159^{*}$ \\
Pérdida de apetito &,- 028 &,- 035 &,- 075 &,- 092 \\
Estreñimiento &,- 052 &,- 108 &,- 060 &, 029 \\
Diarrea &,- 087 &,- 077 &,$- 153^{*}$ &,$- 181^{*}$ \\
Dificultades económicas &,- 079 &,- 064 &,- 027 &,- 103 \\
\hline Escala de estrés percibido &,$- 262^{* *}$ &,$- 243^{* *}$ &,$- 264^{* *}$ &,$- 562^{* *}$ \\
\hline
\end{tabular}

Nota. ${ }^{* *} p \leq 0,01 ; * p \leq 0,05$.

\section{Modelos predictivos de la calidad de vida y el estrés percibido}

Con el objetivo de conocer los posibles factores predictores de las distintas dimensiones de la calidad de vida y del estrés percibido se realizaron análisis de regresión. En cada una de las ecuaciones se usa una de las siguientes variables dependientes: estado global de salud, área de funcionamiento, área de síntomas y estrés percibido. Como predictores, en cada ecuación, usamos las variables de satisfacción con el apoyo recibido emocional, instrumental e informacional del personal sanitario y el optimismo disposicional (que han resultado significativas en el análisis de correlación), junto a variables sociodemográficas y médicas que han resultado significativas en los análisis realizados. Los modelos de regresión finales para cada variable dependiente se muestran en la tabla 6 y 7 .

Tabla 6. Análisis de regresión lineal múltiple para estrés percibido, estado global de salud y escalas funcionales $(n=200)$

\begin{tabular}{|c|c|c|c|c|c|}
\hline \multicolumn{6}{|l|}{ Estrés percibido } \\
\hline \multirow[t]{2}{*}{ Modelo } & \multicolumn{2}{|c|}{ Coeficientes no estandarizados } & $\begin{array}{l}\text { Coeficientes } \\
\text { estandarizados }\end{array}$ & $t$ & Sig. \\
\hline & $\mathrm{B}$ & Error estándar & Beta & & \\
\hline (constante) & 80,947 & 4,547 & & 17,801 & $<, 001 * *$ \\
\hline Satisfacción apoyo instrumental & $-2,673$ & ,783 &,- 233 & $-3,414$ &, $001 * *$ \\
\hline Optimismo &,- 468 & 061 &,- 524 & $-7,664$ & $<, 001 * *$ \\
\hline \multicolumn{6}{|c|}{$R=, 608 R^{2}=, 369 R^{2}$ ajustado $=, 360 F=40,681 \mathrm{Sig}=<, 001 * *$} \\
\hline \multicolumn{6}{|c|}{ Estado global de salud } \\
\hline \multirow[t]{2}{*}{ Modelo } & \multicolumn{2}{|c|}{ Coeficientes no estandarizados } & $\begin{array}{l}\text { Coeficientes } \\
\text { estandarizados }\end{array}$ & $t$ & Sig. \\
\hline & $\mathrm{B}$ & Error estándar & Beta & & \\
\hline (constante) & 55,372 & 11,263 & & 4,916 & $<, 001 * *$ \\
\hline Satisfacción apoyo informacional & 3,129 & 1,302 & ,207 & 2,404 &, $018^{*}$ \\
\hline Optimismo & ,336 & ,096 &, 279 & 3,506 &, $001 * *$ \\
\hline Género & $-9,885$ & 4,360 &,- 191 & $-2,267$ &, $025^{*}$ \\
\hline Fase del proceso médico & $-14,146$ & 3,965 &,- 324 & $-3,567$ & $<, 001 * *$ \\
\hline \multicolumn{6}{|c|}{$R=, 416 R^{2}=, 173 R^{2}$ ajustado $=, 149 F=7,163 \mathrm{Sig}=<, 001 * *$} \\
\hline
\end{tabular}




\begin{tabular}{|c|c|c|c|c|c|}
\hline \multicolumn{6}{|l|}{ Funcionamiento físico } \\
\hline \multirow[t]{2}{*}{ Modelo } & \multicolumn{2}{|c|}{ Coeficientes no estandarizados } & $\begin{array}{c}\text { Coeficientes } \\
\text { estandarizados }\end{array}$ & $t$ & Sig. \\
\hline & $\mathrm{B}$ & Error estándar & Beta & & \\
\hline (constante) & 103,817 & 4,826 & & 21,513 & $<, 001 * *$ \\
\hline Género & $-7,897$ & 2,620 &,- 207 & $-3,015$ &, $003 * *$ \\
\hline Fase del proceso médico & $-12,138$ & 2,280 &,,,- 353 & $-5,325$ & $<, 001 * *$ \\
\hline Hormonoterapia & $-6,473$ & 2,784 &,- 156 & $-2,325$ &, $021^{*}$ \\
\hline \multicolumn{6}{|c|}{$R=, 420 R^{2}=, 177 R^{2}$ ajustado $=, 164 F=13,949 \mathrm{Sig}=<, 001^{* *}$} \\
\hline \multicolumn{6}{|c|}{ Funcionamiento de rol } \\
\hline \multirow[t]{2}{*}{ Modelo } & \multicolumn{2}{|c|}{ Coeficientes no estandarizados } & $\begin{array}{l}\text { Coeficientes } \\
\text { estandarizados }\end{array}$ & $t$ & Sig. \\
\hline & $\mathrm{B}$ & Error estándar & Beta & & \\
\hline (constante) & 89,772 & 13,993 & & 6,416 & $<, 001 * *$ \\
\hline Optimismo & ,330 &, 127 & ,211 & 2,593 &, $011^{*}$ \\
\hline Género & $-14,798$ & 5,807 &,- 220 & $-2,548$ &, $012 *$ \\
\hline Fase del proceso médico & $-17,586$ & 4,976 &,- 310 & $-3,534$ &, $001 * *$ \\
\hline \multicolumn{6}{|c|}{$R=, 346 R^{2}=, 120 R^{2}$ ajustado $=, 101 F=6,266 \mathrm{Sig}=, 001 * *$} \\
\hline \multicolumn{6}{|c|}{ Funcionamiento emocional } \\
\hline \multirow[t]{2}{*}{ Modelo } & \multicolumn{2}{|c|}{ Coeficientes no estandarizados } & $\begin{array}{c}\text { Coeficientes estan- } \\
\text { darizados }\end{array}$ & $t$ & Sig. \\
\hline & $\mathrm{B}$ & Error estándar & Beta & & \\
\hline (constante) & 24,633 & 7,563 & & 3,257 &, $001 * *$ \\
\hline Satisfacción apoyo emocional & 3,379 & 1,262 & ,205 & 2,677 &, $008 * *$ \\
\hline Optimismo &, 503 &, 103 &, 373 & 4,865 & $<, 001 * *$ \\
\hline \multicolumn{6}{|c|}{$R=, 455 R^{2}=, 207 R^{2}$ ajustado $=, 196 F=18,140 \mathrm{Sig}=<, 001^{* *}$} \\
\hline \multicolumn{6}{|c|}{ Funcionamiento cognitivo } \\
\hline \multirow[t]{2}{*}{ Modelo } & \multicolumn{2}{|c|}{ Coeficientes no estandarizados } & $\begin{array}{c}\text { Coeficientes estan- } \\
\text { darizados }\end{array}$ & $t$ & Sig. \\
\hline & B & Error estándar & Beta & & \\
\hline (constante) & 61,593 & 13,143 & & 4,686 & $<, 001 * *$ \\
\hline Satisfacción apoyo instrumental & 4,303 & 1,459 &, 241 & 2,950 &, $004 * *$ \\
\hline Optimismo & ,291 &, 108 & ,209 & 2,689 &, $008 * *$ \\
\hline Género & $-11,207$ & 4,821 &,- 188 & $-2,324$ &, $022 *$ \\
\hline \multicolumn{6}{|c|}{$R=, 434 R^{2}=, 188 R^{2}$ ajustado $=, 171 F=10,683 \mathrm{Sig}=<, 001^{* *}$} \\
\hline \multicolumn{6}{|c|}{ Funcionamiento social } \\
\hline \multirow[t]{2}{*}{ Modelo } & \multicolumn{2}{|c|}{ Coeficientes no estandarizados } & $\begin{array}{l}\text { Coeficientes estan- } \\
\text { darizados }\end{array}$ & $t$ & Sig. \\
\hline & $\mathrm{B}$ & Error estándar & Beta & & \\
\hline (constante) & 43,853 & 8,624 & & 5,085 & $<, 001 * *$ \\
\hline Optimismo & 437 &, 131 & 271 & 3,332 &, $001 * *$ \\
\hline$R=, 271 R^{2}=, 073 R^{2}$ ajustado $=$, & $7 F=11,1$ & $\operatorname{Sig}=, 001^{* *}$ & & & \\
\hline
\end{tabular}

Nota 1. **p $\leq 0,01 ; * p \leq 0,05$

Nota 2. Género: 1 = hombre y $2=$ mujer; Fase proceso médico: $0=$ En revisión y $1=$ En tratamiento; Hormonoterapia: 0 $=$ No y $1=\mathrm{Si}$ 
Tabla 7. Análisis de regresión lineal múltiple para escalas / ítems de síntomas $(\mathrm{n}=200)$

\begin{tabular}{|c|c|c|c|c|c|}
\hline \multicolumn{6}{|l|}{ Fatiga } \\
\hline \multirow[t]{2}{*}{ Modelo } & \multicolumn{2}{|c|}{ Coeficientes no estandarizados } & Coeficientes & $t$ & Sig. \\
\hline & $\mathrm{B}$ & Error estándar & $\begin{array}{l}\text { estandarizados } \\
\text { Beta }\end{array}$ & & \\
\hline (constante) & 45,843 & 14,279 & & 3,210 &, $002 * *$ \\
\hline Satisfacción apoyo instrumental & $-3,898$ & 1,650 &,- 206 & $-2,363$ &, $020^{*}$ \\
\hline Optimismo &,- 395 &, 117 &,- 267 & $-3,370$ &, $001 * *$ \\
\hline Género & 14,696 & 5,405 & ,232 & 2,719 & $007 * *$ \\
\hline Fase del proceso médico & 12,451 & 4,804 & 233 & 2,592 &, $011 *$ \\
\hline \multicolumn{6}{|c|}{$\begin{array}{l}R=, 419 R^{2}=, 176 R^{2} \text { ajustado }=, 152 F=7,311 \mathrm{Sig}=<, 001 * * \\
\text { Dolor }\end{array}$} \\
\hline \multirow[t]{2}{*}{ Modelo } & \multicolumn{2}{|c|}{ Coeficientes no estandarizados } & $\begin{array}{l}\text { Coeficientes } \\
\text { estandarizados }\end{array}$ & $t$ & Sig. \\
\hline & $\mathrm{B}$ & Error estándar & Beta & & \\
\hline (constante) & 21,019 & 2,001 & & 10,503 & $<, 001 * *$ \\
\hline Hormonoterapia & 16,282 & 4,356 &, 257 & 3,738 & $<, 001 * *$ \\
\hline \multicolumn{6}{|c|}{$R=, 257 R^{2}=, 066 R^{2}$ ajustado $=, 061 F=13,972 \mathrm{Sig}=<, 001 * *$} \\
\hline \multicolumn{6}{|c|}{ Disnea } \\
\hline \multirow[t]{2}{*}{ Modelo } & \multicolumn{2}{|c|}{ Coeficientes no estandarizados } & Coeficientes & $t$ & Sig. \\
\hline & $\mathrm{B}$ & Error estándar & Beta & & \\
\hline (constante) & 29,111 & 6,780 & & 4,294 & $<, 001 * *$ \\
\hline Optimismo &,- 264 &, 103 &,- 211 & $-2,557$ &, $012 * *$ \\
\hline \multicolumn{6}{|c|}{$R=, 211 R^{2}=, 045 R^{2}$ ajustado $=, 038 F=6,540 \mathrm{Sig}=, 012^{*}$} \\
\hline \multicolumn{6}{|c|}{ Pérdida de apetito } \\
\hline \multirow[t]{2}{*}{ Modelo } & \multicolumn{2}{|c|}{ Coeficientes no estandarizados } & Coeficientes & $t$ & Sig. \\
\hline & $\mathrm{B}$ & Error estándar & Beta & & \\
\hline (constante) & 5,322 & 1,919 & & 2,774 & $<, 001 * *$ \\
\hline Fase del proceso médico & 9,904 & 3,015 & 227 & 3,285 &, $001 * *$ \\
\hline \multicolumn{6}{|c|}{$R=, 227 R^{2}=, 052 R^{2}$ ajustado $=, 047 F=10,792 \mathrm{Sig}=, 001^{*} *$} \\
\hline \multicolumn{6}{|c|}{ Estreñimiento } \\
\hline \multirow[t]{2}{*}{ Modelo } & \multicolumn{2}{|c|}{ Coeficientes no estandarizados } & $\begin{array}{l}\text { Coeficientes } \\
\text { estandarizados }\end{array}$ & $t$ & Sig. \\
\hline & $\mathrm{B}$ & Error estándar & Beta & & \\
\hline (constante) & 22,868 & 2,810 & & 8,139 & $<, 001 * *$ \\
\hline Radioterapia & $-11,069$ & 3,728 &,- 207 & $-2,969$ &, $003 * *$ \\
\hline \multicolumn{6}{|c|}{$R=, 207 R^{2}=, 043 R^{2}$ ajustado $=, 038 F=8,813 \mathrm{Sig}=, 003^{* *}$} \\
\hline \multicolumn{6}{|c|}{ Diarrea } \\
\hline \multirow[t]{2}{*}{ Modelo } & \multicolumn{2}{|c|}{ Coeficientes no estandarizados } & $\begin{array}{c}\text { Coeficientes } \\
\text { estandarizados }\end{array}$ & $t$ & Sig. \\
\hline & $\mathrm{B}$ & Error estándar & Beta & & \\
\hline (constante) & 23,671 & 6,466 & & 3,661 & $<, 001 * *$ \\
\hline Optimismo &,- 262 &, 100 &,- 219 & $-2,632$ &, $009^{* *}$ \\
\hline Fase del proceso médico & 8,811 & 3,606 & ,203 & 2,444 &, $016^{*}$ \\
\hline
\end{tabular}




\begin{tabular}{|c|c|c|c|c|c|}
\hline \multicolumn{6}{|l|}{ Dificultades económicas } \\
\hline \multirow[t]{2}{*}{ Modelo } & \multicolumn{2}{|c|}{ Coeficientes no estandarizados } & $\begin{array}{c}\text { Coeficientes } \\
\text { estandarizados }\end{array}$ & $t$ & Sig. \\
\hline & $\mathrm{B}$ & Error estándar & Beta & & \\
\hline (constante) & 11,839 & 2,934 & & 4,035 & $<, 001 * *$ \\
\hline Fase del proceso médico & 16,533 & 4,248 & ,262 & 3,892 & $<, 001 * *$ \\
\hline Hormonoterapia & 16,784 & 5,114 & ,221 & 3,282 &, $001 * *$ \\
\hline
\end{tabular}

$R=, 338 R^{2}=, 114 R^{2}$ ajustado $=, 105 F=12,618 \mathrm{Sig}=<, 001 * *$

Nota 1. **p $\leq 0,01 ; * p \leq 0,05$

Nota 2. Género: 1 = hombre y $2=$ mujer; Fase proceso médico: $0=$ En revisión y $1=$ En tratamiento; Hormonoterapia: $0=$ No y $1=\mathrm{Si}$; Radioterapia: $0=$ No y $1=\mathrm{Si}$

El análisis de regresión reveló modelos significativos en una parte de la varianza significativa para el estado global de salud, para escalas de funcionamiento, escalas / ítems de síntomas y para estrés percibido. Para el estrés percibido, la ecuación de regresión tiene una $\mathrm{R}^{2}=0,369, \mathrm{~F}=40,681, p<0,001$. La satisfacción con el apoyo instrumental del personal sanitario $(\beta=-0,233, p=0,001)$ y el optimismo $(\beta=$ $-0,524, p<0,001)$ se relaciona con una disminución del estrés percibido en pacientes con cáncer. Para el estado global de salud, la ecuación de regresión tiene una $\mathrm{R}^{2}$ $=0,173, \mathrm{~F}=7,163, p<0,001$. Ser mujer $(\beta=-0,191, p=0,025)$ y encontrarse en tratamiento $(\beta=-0,324, p<0,001)$ contribuye a predecir un peor estado global de salud. En cambio, la satisfacción con el apoyo informacional del personal sanitario $(\beta=0,207, p=0,018)$ y el optimismo disposicional del paciente $(\beta=0,279, p=$ $0,001)$ se relaciona con un mejor estado global de salud. Respecto a las escalas de funcionamiento, para el funcionamiento físico, la ecuación de regresión tiene una $\mathrm{R}^{2}=0,177, \mathrm{~F}=13,949, p<0,001$. Ser mujer $(\beta=-0,207, p=0,003)$, encontrarse en tratamiento $(\beta=-0,353, p<0,001)$ y haber recibido hormonoterapia $(\beta=-0,156$, $p=0,021)$ contribuye a un peor funcionamiento físico. La ecuación de regresión para el funcionamiento de rol tiene una $\mathrm{R}^{2}=0,120, \mathrm{~F}=6,266, p=0,001$. Un mayor optimismo $(\beta=0,211, p=0,011)$, ser hombre $(\beta=-0,220, p=0,012)$, y encontrarse en revisión $(\beta=-0,310, p=0,001)$ contribuye a un mejor funcionamiento de rol. Para el funcionamiento emocional la ecuación de regresión tiene una $\mathrm{R}^{2}=0,207, \mathrm{~F}$ $=18,140, p<0,001$. La satisfacción con el apoyo emocional del personal sanitario $(\beta=0,205, p=0,008)$ y el optimismo $(\beta=0,373, p<0,001)$ contribuye a un mayor funcionamiento de rol. La ecuación de regresión para el funcionamiento cognitivo tiene una $\mathrm{R}^{2}=0,188, \mathrm{~F}=10,683, p<0,001$. La satisfacción con el apoyo instrumental del personal sanitario $(\beta=0,241, p=0,004)$, el optimismo $(\beta=0,209, p=0,008)$ y ser hombre $(\beta=-0,188, p=0,022)$ contribuye a un mejor funcionamiento cognitivo. Respecto al funcionamiento social la ecuación de regresión tiene una $\mathrm{R}^{2}=0,073, \mathrm{~F}$ $=11,104, p=0,001$. En este caso el optimismo $(\beta=0,271, p=0,001)$ contribuye a predecir un mejor funcionamiento social.

Respecto a las escalas de síntomas, la fatiga tiene una $\mathrm{R}^{2}=0,176, \mathrm{~F}=7,311$, $p<0,001$. La satisfacción con el apoyo instrumental del personal sanitario $(\beta=$ $-0,206, p=0,020)$, el optimismo $(\beta=-0,267, p=0,001)$ ser hombre $(\beta=0,232$, $p=0,007)$ y encontrarse en revisión $(\beta=0,233, p=0,011)$ contribuye a predecir una menor fatiga. La ecuación de regresión para el dolor tiene una $\mathrm{R}^{2}=0,066, \mathrm{~F}=$ $13,972, p<0,001$. El haber recibido hormonoterapia $(\beta=0,257, p<0,001)$ predice 
un mayor dolor. Para la disnea la ecuación de regresión tiene una $\mathrm{R}^{2}=0,045, \mathrm{~F}$ $=6,540, p=0,012$. El optimismo $(\beta=-, 211, p=0,012)$ contribuye a una menor disnea. La ecuación de regresión para la pérdida de apetito tiene una $\mathrm{R}^{2}=0,052, \mathrm{~F}$ $=10,792, p=0,001$. En este caso, encontrarse en tratamiento $(\beta=0,227, \mathrm{p}=0,001)$ contribuye a una mayor pérdida de apetito. Para el estreñimiento la ecuación de regresión tiene una $\mathrm{R}^{2}=0,043, \mathrm{~F}=8,813, p=0,003$. Haber recibido radioterapia $(\beta$ $=-0,207, p=0,003)$ contribuye a predecir un menor estreñimiento. La ecuación de regresión para la diarrea tiene una $\mathrm{R}^{2}=0,073, \mathrm{~F}=5,440, p=0,005$. El optimismo $(\beta=-0,219, p=0,009)$ y encontrarse en revisión $(\beta=0,203, p=0,016)$ contribuye a una menor diarrea. Para terminar, la ecuación de regresión para las dificultades económicas tiene una $\mathrm{R}^{2}=0,114, \mathrm{~F}=12,618, p<0,001$. En este caso, encontrarse en tratamiento $(\beta=0,262, p<0,001)$ y haber recibido hormonoterapia $(\beta=0,221$, $p=0,001)$ predice mayores dificultades económicas. En cambio, los resultados no mostraron modelos significativos para predecir las náuseas y vómitos ni para predecir el insomnio en el paciente.

\section{Discusión y conclusión}

El objetivo de este estudio ha sido analizar la relación del apoyo social y el optimismo con la calidad de vida y el estrés percibido en los enfermos oncológicos atendiendo a las diferentes fases de la enfermedad, los tipos de tratamientos y las diferencias de género. Los resultados en general han confirmado nuestras hipótesis.

En relación a la primera hipótesis los resultados han mostrado que las mujeres con cáncer perciben peor estado global de salud, menor funcionamiento, mayores síntomas y mayor estrés que los hombres, estos resultados concuerdan con resultados de diferentes estudios ${ }^{(13)}$. Las principales diferencias se producen entre hombres y mujeres que se encuentran en revisión. En este caso, las mujeres perciben peor calidad de vida y un mayor estrés que los hombres. Hay que recordar que en la fase de revisión se produce una vuelta a la "normalidad" por parte del paciente. Una vuelta a la "normalidad" donde no hay tantos síntomas visibles como pueden darse en la fase de tratamiento, pero el paciente aún puede seguir necesitando determinados apoyos que quizás no sean proporcionados por su círculo social. De este modo la reducción que se produce en la calidad de vida y el aumento del estrés percibido puede darse en mayor medida en las mujeres ya que, aún hoy día, suele existir un predominio de ciertas responsabilidades en las mujeres, como puede ser adquirir el rol de cuidadora de alguna persona con necesidades en su familia ${ }^{(61)}$.

La siguiente hipótesis que planteamos es que las personas que están en tratamiento perciben más estrés y tienen peor calidad de vida. Nuestros resultados muestran que los pacientes que se encuentran actualmente en tratamiento muestran una menor calidad de vida en casi todas sus dimensiones, hecho que parece factible teniendo en cuenta la toxicidad del tratamiento. No encontramos diferencia en cuanto al estrés percibido entre pacientes que se encuentran en tratamiento y pacientes que se encuentran en revisión. Esto es posible ya que todos los pacientes se enfrentan a dificultades que se dan en todas las fases de la enfermedad. Incluso esas dificultades pueden iniciarse en la fase de tratamiento y continuar en la fase de revisión. Se pueden dar situaciones que funcionan como una fuente potencial de estrés como la ruptura de relaciones o la interrupción del trabajo ${ }^{(17)}$. Además en la fase de revisión 
existe un miedo a la posible recidiva ${ }^{(22)}$ que aumenta el estrés y reduce el bienestar de los sobrevivientes de cáncer ${ }^{(23)}$. Respecto al tipo de tratamiento recibido, llama la atención que las personas que han recibido quimioterapia perciben un menor nivel de estrés que las personas que no han recibido este tratamiento. Una posible explicación, como apuntan algunos autores ${ }^{(62)}$ puede deberse a que la quimioterapia es el tratamiento "prototipo" respecto al cáncer, es la primera imagen y el primer miedo que puede sentir una persona a la que le diagnostican cáncer. El miedo, la ansiedad y la angustia durante el tratamiento quimioterápico y el seguimiento posterior pueden reducirse una vez recibida la información necesaria. En cambio, las personas que han recibido tratamiento hormonal perciben una peor calidad de vida y un mayor estrés que las personas que no han tenido este tipo de tratamiento. De hecho, el haber recibido tratamiento hormonoterápico llega a predecir un aumento de los síntomas. Hay que tener en cuenta que el tratamiento hormonal suele tener mayor duración que otro tipo de tratamientos, incluso años. Aunque, en general se asocie la hormonoterapia con menores efectos adversos que otros tratamientos, también tiene importantes efectos negativos sobre los pacientes influyendo negativamente sobre la calidad de vida y el estrés de los pacientes durante largos intervalos de tiempo ${ }^{(63)}$.

En la siguiente hipótesis planteamos que la satisfacción con el apoyo proporcionado por el personal sanitario y el optimismo están relacionados positivamente con la calidad de vida y negativamente con el estrés percibido en pacientes con cáncer, hipótesis que se confirma con los resultados obtenidos. El apoyo proporcionado por el personal sanitario ayuda a los pacientes a sentirse con confianza en la situación en la que se encuentran ${ }^{(64)}$. Además ayuda a normalizar la angustia de los pacientes ${ }^{(65)}$ pudiendo aliviar la ansiedad. El apoyo social de los profesionales sanitarios ayuda a mejorar los sentimientos de bienestar y el afrontamiento en los pacientes ${ }^{(66)}$ y esto ayuda a percibir un menor estrés. Los resultados coinciden con estudios en los que el optimismo se relaciona con una mayor calidad de $\operatorname{vida}^{(30)}$ asociándose con una disminución de los síntomas ${ }^{(33)}$ y con una reducción del estrés percibido ${ }^{(35)}$ ya que el optimismo es una herramienta para reducir los eventos que producen estrés en el paciente ${ }^{(36)}$. Los resultados muestran que no existe relación entre la satisfacción con el apoyo social proporcionado por el personal sanitario y las dificultades económicas. Es posible que el apoyo del personal sanitario solo repercuta en los aspectos vinculados a la enfermedad pero que no guarden relación alguna con problemas colaterales como las circunstancias económicas de los pacientes.

En relación a los tipos de apoyo que son más importantes para la calidad de vida y la menor percepción de estrés, hemos encontrado que cada tipo de apoyo (emocional, instrumental o informacional) proporcionado por el personal sanitario guarda relación con algún aspecto de la calidad de vida o la percepción de estrés. Parece que tener una información adecuada de la enfermedad, los tratamientos y resolver dudas con el personal sanitario tiene un efecto importante en la calidad de vida de los pacientes ${ }^{(48)}$. Este tipo de apoyo proporcionado por el personal sanitario ayuda al paciente a tener información explícita sobre su enfermedad, tratamiento, pronóstico y efectos secundarios que ayuda a su mejor ajuste a la enfermedad ${ }^{(49)}$ ya que los pacientes necesitan información adaptada a sus necesidades para así poder reducir sus síntomas de modo adecuado. Nuestro estudio también refleja cómo la satisfacción con el apoyo instrumental proporcionado por el personal sanitario es un predictor del aumento de algunos indicadores de la calidad de vida y contribuye a disminuir el estrés. Es decir, si el paciente se encuentra satisfecho con los cuidados recibidos por el personal sanitario 
tenderá a reducir su percepción de estrés ante la enfermedad. Este tipo de apoyo ha sido escasamente estudiado y sin embargo hemos encontrado efectos positivos sobre el estrés de los pacientes. En nuestro estudio también hemos encontrado resultados significativos del apoyo emocional del personal sanitario como variable predictora, coincidiendo con estudios que destacan la importancia del mismo en relación a un mejor ajuste a la enfermedad reduciendo el estrés percibido ${ }^{(48)}$.

Hay que destacar que la mayoría de los estudios no diferencian entre los tres tipos de apoyo y en nuestro estudio hemos podido confirmar la importancia del apoyo emocional, informacional e instrumental en la calidad de vida y el estrés de los pacientes oncológicos. Lo que muestra la importancia de conocer las necesidades de los pacientes para prestar el apoyo que realmente necesitan.

Esta información es muy importante para diseñar programas de atención a los pacientes de cáncer basándonos en la satisfacción con el apoyo proporcionado del personal sanitario. Hay que tener en cuenta que los pacientes le dan un alto valor al apoyo proporcionado por el personal sanitario, similar al de familiares y amigos ${ }^{(64)}$ siendo una de la fuentes de apoyo más importante durante la fase de diagnóstico y tratamiento. Es importante dar a conocer al personal sanitario la importancia de proporcionar apoyo al paciente a lo largo de toda la trayectoria del proceso sanitario del cáncer y de ser activos en el modo de proporcionar este apoyo ${ }^{(67)}$.

\section{Limitaciones del estudio}

En este estudio las asociaciones de mujeres son las que más han podido participar, por ello hay una menor cantidad en nuestro estudio de pacientes varones. Para futuros estudios se podría aumentar el número de hombres y el número de pacientes. Otra propuesta para futuros estudios es analizar los efectos del apoyo social proporcionado por el personal sanitario atendiendo al tipo de cáncer.

\section{Implicaciones clínicas}

Es de real importancia estudiar los aspectos psicológicos de los pacientes con cáncer ya que no solo el objetivo debe ser aumentar su cantidad de vida, sino que también es muy importante aumentar la calidad de la misma durante el proceso de afrontamiento al cáncer. El incremento de la expectativa de vida debe ir acompañada de un menor deterioro en su calidad de vida y menor percepción de estrés. Se podrían diseñar estrategias de intervención más eficaces para determinar el beneficio global en la calidad de vida del paciente, partiendo de un análisis de las necesidades específicas de apoyo en relación al ámbito sanitario. Ya que como se ha comprobado en este estudio tienen un papel fundamental en la calidad de vida de los pacientes. Es importante que la intervención se centre en mejorar el optimismo de los pacientes. Esta mejora puede llevarse a cabo a través de ciertas estrategias como proporcionarle por parte del personal sanitario información veraz sobre la enfermedad y el proceso médico, con el fin de que el paciente tenga unas expectativas favorables respecto a su futuro, disminuyendo así el miedo y ansiedad que produce el ser diagnosticado de cáncer. De este modo, se favorecerá que el paciente se adapte mejor a la enfermedad y mejore su calidad de vida. A raíz de los resultados obtenidos se resalta la importancia de llevar a cabo intervenciones con los profesionales de la salud para hacerles conscientes de lo importante que es su apoyo para la salud y calidad de vida de los enfermos de cáncer. 


\section{Agradecimientos}

Los autores quieren agradecer su colaboración en este estudio al Hospital Costa del Sol (Marbella, Málaga, España) y a todas las asociaciones y pacientes que participaron en el mismo. Este trabajo ha sido apoyado por los Grupos PAIDI, Junta de Andalucía, España [HUM-590].

\section{Referencias bibliográficas}

1. World Health Organization. Cancer [Internet]. 2018 [Acceso 5 de julio de 2020]. Disponible en: http://www.who.int/news-room/fact-sheets/detail/cancer

2. World Health Organization. Regional Office for Europe. Cancer [Internet]. [Acceso 6 de julio de 2020]. Disponible en: http://www.euro.who.int/en/health-topics/noncommunicablediseases/cancer/cancer

3. Instituto Nacional de Estadística. Fallecidos por cáncer en España [Internet]. Instituto Nacional de Estadística. 2019. Disponible en: http://www.ine.es/infografias/infografia fallecidos_cancer.pdf

4. Labrell F, Câmara-Costa H, Dufour C, Grill J, Chevignard M. Maternal stress and pediatric brain cancer: a French study. J Psychosoc Oncol 2019; 37: 96-109. doi:10.1080 /07347332.2018.1529009

5. Rowland JH, Bellizzi KM. Cancer survivorship issues: Life after treatment and implications for an aging population. J Clin Oncol 2014; 32: 2662-8. doi:10.1200/ JCO.2014.55.8361

6. Cella DF. Quality of life: concepts and definition. J Pain Symptom Manage 1994; 9: 18692. doi:10.1016/0885-3924(94)90129-5

7. Pirri C, Bayliss E, Trotter J, Olver IN, Katris P, Drummond P, et al. Nausea still the poor relation in antiemetic therapy? the impact on cancer patients' quality of life and psychological adjustment of nausea, vomiting and appetite loss, individually and concurrently as part of a symptom cluster. Support Care Cancer 2013; 21: 735-48. doi:10.1007/s00520-012-1574-9

8. Gargantini ACG, Martín Casari L. Imagen Corporal y su Influencia en la Calidad de Vida en mujeres con mastectomía. Comparación entre mujeres con reconstrucción inmediata, reconstrucción diferida o sin reconstrucción. Psicooncología 2019; 16: 43-60. doi:10.5209/PSIC.63647

9. Barbus E, Pestean C, Larg MI, Gabora K, Cheptea M, Bonci EA, et al. Quality of life in thyroid cancer: A questionnaire based-study. J Evid Based Psychother 2018; 18: 1-20. doi:10.24193/jebp.2018.1.1

10. Adamowicz K, Janiszewska J, Lichodziejewska-Niemierko M. Prognostic value of patient knowledge of cancer on quality of life in advanced lung cancer during chemotherapy. $\mathrm{J}$ Cancer Educ 2020; 35: 93-9. doi:10.1007/s13187-018-1444-3

11. Lopes AC, Bacalhau R, Santos M, Pereira M, Pereira MG. Contribution of sociodemographic, clinical and psychological variables to quality of life in women with cervical cancer in the follow-up phase. J Clin Psychol Med Settings 2019; 27:603-14. doi:10.1007/s10880-019-09644-0

12. Willems RA, Bolman CAW, Mesters I, Kanera IM, Beaulen AAJM, Lechner L. Cancer survivors in the first year after treatment: The prevalence and correlates of unmet needs in different domains. Psychooncology 2016; 25: 51-7. doi:10.1002/pon.3870 
13. Thomé B, Hallberg IR. Quality of life in older people with cancer - A gender perspective. Eur J Cancer Care 2004; 13: 454-63. doi:10.1111/j.1365-2354.2004.00471.x

14. Franceschi S, La Vecchia C. Cancer epidemiology in the elderly. Crit Rev Oncol Hematol 2001; 39: 219-26. doi:10.1016/S1040-8428(01)00102-0

15. Ezat WS, Noraziani K, Sabrizan O. Improving quality of life among cancer patients in Malaysia. Asian Pac J Cancer Prev 2012; 13: 1069-75. doi:10.7314/APJCP.2012.13.3.1069

16. Puente-Maestu L, Villar F. Implicaciones de la calidad de vida en las decisiones quirúrgicas del cáncer de pulmón. Psicooncologia 2006; 3: 393-405.

17. Fitch M, Longo CJ. Exploring the impact of out-of-pocket costs on the quality of life of Canadian cancer patients. J Psychosoc Oncol 2018; 36: 582-96. doi:10.1080/07347332.2 018.1486937

18. Abdollahi A, Panahipour H, Hosseinian S, Allen KA. The effects of perceived stress on hope in women with breast cancer and the role of psychological hardiness. Psychooncology 2019; 28: 1477-82. doi:10.1002/pon.5102

19. Pedersen AF, Rossen P, Olesen F, Von Der Maase H, Vedsted P. Fear of recurrence and causal attributions in long-term survivors of testicular cancer. Psychooncology 2012; 21: 1222-8. doi:10.1002/pon.2030

20. Miaskowski C, Paul SM, Mastick J, Abrams G, Topp K, Smoot B, et al. Associations between perceived stress and chemotherapy-induced peripheral neuropathy and otoxicity in adult cancer survivors. J Pain Symptom Manage 2018; 56: 88-97. doi:10.1016/j. jpainsymman.2018.02.021

21. Reich RR, Lengacher CA, Alinat CB, Kip KE, Paterson C, Ramesar S, et al. MindfulnessBased Stress Reduction in post-treatment breast cancer patients: immediate and sustained effects across multiple symptom clusters. J Pain Symptom Manage 2017; 53: 85-95. doi:10.1016/j.jpainsymman.2016.08.005

22. Dunn LB, Langford DJ, Paul SM, Berman MB, Shumay DM, Kober K, et al. Trajectories of fear of recurrence in women with breast cancer. Support Care Cancer 2015; 23: 203343. doi:10.1007/s00520-014-2513-8

23. Smith AB, Butow P, Olver I, Luckett T, Grimison P, Toner GC, et al. The prevalence, severity, and correlates of psychological distress and impaired health-related quality of life following treatment for testicular cancer: a survivorship study. J Cancer Surviv 2016; 10: 223-33. doi:10.1007/s11764-015-0468-5

24. Fleer J, Sleijfer D, Hoekstra H, Tuinman M, Klip E, Hoekstra-Weebers J. Objective and subjective predictors of cancer-related stress symptoms in testicular cancer survivors. Patient Educ Couns 2006; 64: 142-50. doi:10.1016/j.pec.2005.12.009

25. Kreitler S, Peleg D, Ehrenfeld M. Stress, self-efficacy and quality of life in cancer patients. Psychooncology 2007; 16: 329-41. doi:10.1002/pon.1063

26. Alcorso J, Sherman KA. Factors associated with psychological distress in women with breast cancer-related lymphoedema. Psychooncology 2016; 25: 865-72. doi:10.1002/pon.4021

27. Carver CS, Scheier MF. Dispositional optimism. Trends Cogn Sci 2014; 18: 293-9. doi:10.1016/j.tics.2014.02.003

28. Saboonchi F, Petersson L, Alexanderson K, Bränström R, Wennman-Larsen A. Expecting the best and being prepared for the worst: structure, profiles, and 2-year temporal stability of dispositional optimism in women with breast cancer. Psychooncology 2016; 25: 95763. doi:10.1002/pon.4045

29. Finck C, Barradas S, Zenger M, Hinz A. Quality of life in breast cancer patients: associations with optimism and social support. Int J Clin Health Psychol 2018; 18: 27-34. doi:10.1016/j.ijchp.2017.11.002 
30. Mera PC, Ortiz M. La relación del optimismo y las estrategias de afrontamiento con la calidad de vida de mujeres con cáncer de mama. Ter Psicol 2012; 30: 69-78. doi:10.4067/ S0718-48082012000300007

31. Schou I, Ekeberg Ø, Ruland CM, Sandvik L, Kåresen R. Pessimism as a predictor of emotional morbidity one year following breast cancer surgery. Psychooncology 2004; 13 : 309-20. doi:10.1002/pon.747

32. Jaime-Bernal L, Juárez-García DM, Téllez A. La influencia del estrés y el optimismo sobre la calidad de sueño de mujeres con cáncer de mama. Psicooncología 2018; 15: 24960. doi:10.5209/PSIC.61434

33. Lemola S, Räikkönen K, Gomez V, Allemand M. Optimism and self-esteem are related to sleep. Results from a large community-based sample. Int J Behav Med 2013; 20: 567-71. doi:10.1007/s12529-012-9272-Z

34. Schou I, Ekeberg O, Sandvik L, Ruland CM. Stability in optimism-pessimism in relation to bad news: A study of women with breast cancer. J Pers Assess 2005; 84: 148-54. doi:10.1207/s15327752jpa8402_04

35. Trunzo JJ, Pinto BM. Social support as a mediator of optimism and distress in breast cancer survivors. J Consult Clin Psychol 2003; 71: 805-11. doi:10.1037/0022-006X.71.4.805

36. Shaheen N, Andleeb S, Ahmad S, Bano A. Effect of optimism on psychological stress in breast cancer women. J Soc Sci 2014; 8: 84-90.

37. Sephton S, Spiegel D. Circadian disruption in cancer: a neuroendocrine-immune pathway from stress to disease? Brain Behav Immun 2003; 17: 321-8. doi:10.1016/S08891591(03)00078-3

38. Durá-Ferrandis E, Saéz-Mansilla C, Murphy SE, Johnson AC, Tercyak KP. Actitudes hacia la maternidad y el apoyo social como predictores de trastornos emocionales en mujeres jóvenes con cáncer recientemente diagnosticadas que se someten a la preservación de la fertilidad. Psicooncología 2019; 16: 127-41. doi:10.5209/PSIC.63652

39. Villanova Quiroga C, Fritzen Binfaré L, Rudnicki T, Iracema de Lima Argimon I. Ruminación y apoyo social como predictores del crecimiento postraumático en mujeres con cáncer de mama: una revisión sistemática. Psicooncología 2018; 15: 301-14. doi:10.5209/PSIC.61437

40. Melguizo-Garín A, Martos-Méndez MJ, Hombrados-Mendieta I. Influencia del apoyo social sobre el estrés y la satisfacción vital en padres de niños con cáncer desde una perspectiva multidimensional. Psicooncología 2019; 16: 25-42. doi:10.5209/PSIC.63646

41. Melguizo-Garín A, Hombrados-Mendieta I, Martos-Méndez MJ. La experiencia de un grupo de apoyo en el proceso de duelo de familiares de niños con cáncer. Un estudio cualitativo. Psicooncología 2020; 17: 117-29. doi:10.5209/psic.68245

42. Lin N, Dean A, Ensel W, editors. Social support, life events and depression. New York: Academic Press, 1986.

43. Breuer N, Sender A, Daneck L, Mentschke L, Leuteritz K, Friedrich M, et al. How do young adults with cancer perceive social support? A qualitative study. J Psychosoc Oncol 2017; 35: 292-308. doi:10.1080/07347332.2017.1289290

44. Taylor SE. Health Psychology. New York: McGraw-Hill; 2006.

45. Lin N. Conceptualizing social support. En: Lin N, Dean A, Ensel W, editors. Social support, life events and depression. New York: Academic Press; 1986. p. 17-30.

46. Thoits PA. Conceptual, methodological, and theoretical problems in studying social support as a buffer against life stress. J Health Soc Behav 1982; 23: 145-59. doi:10.2307/2136511

47. Broadbent E, Petrie KJ, Main J, Weinman J. The Brief Illness Perception Questionnaire. J Psychosom Res 2006; 60: 631-7. doi:10.1016/j.jpsychores.2005.10.020 
48. Drageset S, Lindstrøm TC, Giske T, Underlid K. "The support I need": women's experiences of social support after having received breast cancer diagnosis and awaiting surgery. Cancer Nurs 2012; 35: E39-47. doi:10.1097/NCC.0b013e31823634aa

49. Drageset S, Lindstrøm TC, Giske T, Underlid K. Women's experiences of social support during the first year following primary breast cancer surgery. Scand J Caring Sci 2016; 30: 340-8. doi: $10.1111 / \mathrm{scs} .12250$

50. Linden W, Vodermaier A. Mismatch of desired versus perceived social support and associated levels of anxiety and depression in newly diagnosed cancer patients. Support Care Cancer 2012; 20: 1449-56. doi:10.1007/s00520-011-1228-3

51. Hombrados-Mendieta I, García-Martín MA, Gómez-Jacinto L. The relationship between social support, loneliness, and subjective well-being in a Spanish sample from a multidimensional perspective. Soc Indic Res 2013; 114: 1013-34. doi:10.1007/s11205012-0187-5

52. Aaronson NK, Ahmedzai S, Bergman B, Bullinger M, Cull A, Duez NJ, et al. The European Organization for Research and Treatment of Cancer QLQ-C30: A quality-of-life instrument for use in international clinical trials in oncology. J Natl Cancer Inst 1993; 85: 365-76.

53. Arrarás JI, Illarramendi JJ, Valerdi JJ. El cuestionario de calidad de vida para cáncer de la EORTC, QLQ-C30. Estudio estadístico de validación con una muestra española. Rev Psicol la Salud 1995; 7: 13-33.

54. García-Martín MÁ, Hombrados-Mendieta I, Gómez-Jacinto L. A multidimensional approach to social support: the Questionnaire on the Frequency of and Satisfaction with Social Support (QFSSS). An Psicol 2016; 32: 501-15. doi:10.6018/analesps.32.2.201941

55. Hombrados-Mendieta I, Gómez Jacinto L, Domínguez-Fuentes JM, García-Leiva P, Castro-Travé M. Types of social support provided by parents, teachers and classmates during adolescence. J Community Psychol 2012; 40: 645-664. doi:10.1002/jcop.20523

56. Hombrados-Mendieta I, Millán-Franco M, Gómez-Jacinto L, González-Castro F, MartosMéndez MJ, García-Cid A. Positive influences of social support on sense of community, life satisfaction and the health of immigrants in Spain. Front Psychol 2019; 10: 2555. doi:10.3389/fpsyg.2019.02555.

57. Cohen S, Kamarck T, Mermelstein R. A global measure of perceived stress. J Health Soc Behav 1983; 24: 385. doi:10.2307/2136404

58. Remor E. Psychometric properties of a European Spanish version of the Perceived Stress Scale (PSS). Span J Psychol 2006; 9: 86-93. doi:10.1017/S1138741600006004

59. Scheier MF, Carver CS, Bridges MW. Distinguishing optimism from neuroticism (and trait anxiety, self-mastery, and self-esteem): A reevaluation of the Life Orientation Test. J Pers Soc Psychol 1994; 67: 1063-78. doi:10.1037/0022-3514.67.6.1063

60. Otero JM, Luengo A, Romero E, Gómez JA, Castro C. Psicología de la personalidad. Manual de prácticas. Barcelona: Ariel Practicum, 1998.

61. García-Calvente M del M, Mateo-Rodríguez I, Maroto-Navarro G. El impacto de cuidar en la salud y la calidad de vida de las mujeres. Gac Sanit 2004; 18(Supl.2): 83-92.

62. Trask PC, Paterson AG, Fardig J, Smith DC. Course of distress and quality of life in testicular cancer patients before, during, and after chemotherapy: Results of a pilot study. Psychooncology 2003;12:814-20. doi: 10.1002/pon.703

63. Oraá-Tabernero N, Cruzado JA. Ansiedad y depresión en hombres con cáncer de próstata en función del tipo de tratamiento y su relación con la calidad de vida y la información recibida. Psicooncología 2019; 16: 329-44. doi:10.5209/psic.65594 
64. Sjölander C, Berterö C. The significance of social support and social networks among newly diagnosed lung cancer patients in Sweden. Nurs Health Sci 2008; 10: 182-7. doi:10.1111/j.1442-2018.2008.00395.x

65. Costa-Requena G, Ballester Arnal R, Gil F. Perceived social support in spanish cancer outpatients with psychiatric disorder. Stress Health 2013; 29: 421-6. doi:10.1002/smi.2488

66. Landmark BT, Strandmark M, Wahl A. Breast cancer and experiences of social support: In-depth interviews of 10 women with newly diagnosed breast cancer. Scand J Caring Sci 2002; 16: 216-23. doi:10.1046/j.1471-6712.2002.00059.x

67. Curtis R, Groarke AM, McSharry J, Kerin M. Experience of breast cancer: Burden, benefit, or both? Cancer Nurs 2014; 37: E21-E30. doi:10.1097/NCC.0b013e3182894198 